\title{
Addition of Tethered Nonaromatic Carbon Nucleophiles to \\ Chemoselectively Activated Amides
}

\author{
Guillaume Bélanger, ${ }^{*}$ Robin Larouche-Gauthier, Frédéric Ménard, \\ Miguel Nantel, ${ }^{\dagger}$ and Francis Barabé
}

Laboratoire de synthèse organique et de développement de stratégies de synthèse, Département de Chimie, Université de Sherbrooke, 2500 boulevard Université, Sherbrooke, Québec J1K 2R1, Canada

guillaume.belanger@usherbrooke.ca

Received July 13, 2005

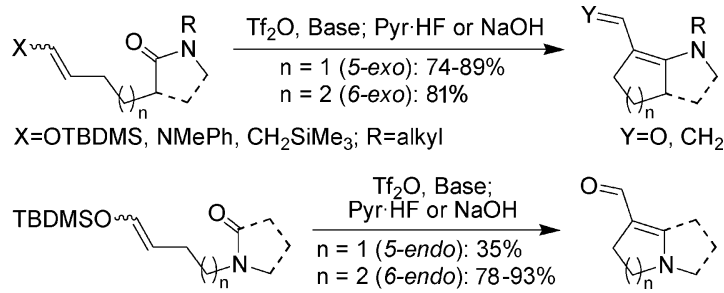

In an effort to develop new ways of synthesizing polycyclic alkaloids, we successfully added silyl enol ethers, allylsilanes, and enamines to iminium ions generated from amides. Because of their higher oxidation state, such iminiums show a yet unexploited advantage of potential double cyclizations over standard Mannich monocyclizations. We report herein the first example of tethered nonaromatic carbon nucleophiles adding to activated amides for the generation of enaminals of various ring sizes, with endo- or exo-cyclic nitrogen.

Numerous efficient approaches to construct biologically active polycyclic alkaloids use intramolecular Mannich addition to an iminium ion. ${ }^{1}$ These iminium ions usually bear a carbon of oxidation state II (two $C$-heteroatom bonds), due to the ways they are usually generated, ${ }^{1 b, 2}$ inherently limiting the approach to a monocyclization. Our goal is to exploit a higher level of oxidation state by generating iminium ions from amides. ${ }^{3}$ With nucleophiles tethered to the amide (e.g., 1, Scheme 1), we would have an access to polycyclic alkaloid

† Current address: MDS Pharma Services, 2350 rue Cohen, St-Laurent (Montréal), Québec H4R 2N6, Canada.

(1) (a) Iminium Salts in Organic Chemistry; Böhme, H., Viehe, H. G., Eds.; Wiley: New York, 1976 (part I); 1979 (part 2). (b) Overman, L. E.; Ricca, D. J. In Comprehensive Organic Synthesis; Trost, B. M., Flemming, I., Heathcock, C. H., Eds; Pergamon: Oxford, 1991; Vol. 2, p 1007.

(2) (a) Arend, M.; Westermann, B.; Risch, N. Angew. Chem., Int. Ed. 1998, 37, 1044. (b) Royer, J.; Bonin, M.; Micouin, L. Chem. Rev. 2004, 104, 2311.

(3) The next level of oxidation state would be carbamates or ureas. For a precise example using carbamates, see: Magnus, P.; Gazzard, L.; Hobson, L.; Payne, A. H.; Rainey, T. J.; Westlund, N.; Lynch, V. Tetrahedron 2002 58,3423 . This work was published while we were already engaged in our studies with amides.

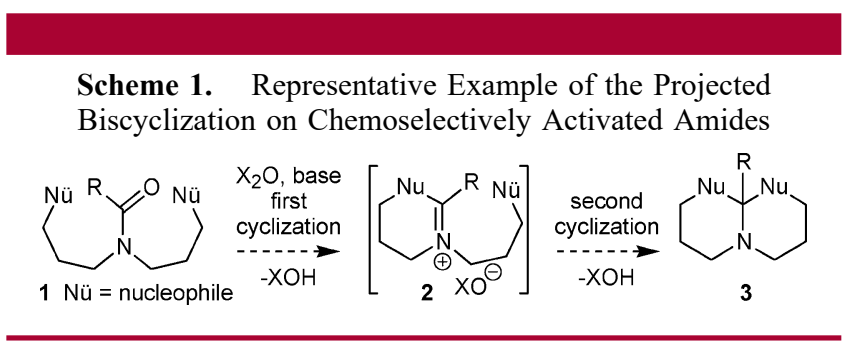

skeletons in one synthetic step from linear precursors through novel sequential nucleophilic cyclizations on the same amide carbon, ${ }^{4}$ thus creating a tertiary $(3, \mathrm{R}=\mathrm{H})$ or a quaternary $(3, \mathrm{R}=$ alkyl) center $\alpha$ to nitrogen, as found in the skeleton of a myriad of alkaloids. Since the ways to attach the nucleophiles to the amide are diverse ( $C$ - and/or $N$-bound), various complex alkaloid skeletons could be prepared, thus

(4) For selected examples of sequential iminium cyclizations, although not performed on the same carbon, see: (a) Jackson, A. H.; Shannon, P. V. R.; Wilkins, D. J. Tetrahedron Lett. 1987, 28, 4901. (b) He, F.; Bo, Y.; Altom, D.; Corey, E. J. J. Am. Chem. Soc. 1999, 121, 6771. 
presenting a great versatility through rapid increase of complexity from easily assembled substrates.

The only reported examples of tethered carbon nucleophiles adding to iminium ions generated from amides ${ }^{5}$ are either indoles ${ }^{6}$ or activated benzene rings. ${ }^{7}$ We wanted to find out if tethered nonaromatic carbon nucleophiles would participate in the cyclization. This however presents a serious difficulty: the usual amide activation conditions involve Lewis or Brønsted acids that react with most nucleophiles. ${ }^{8}$ This paper summarizes the successful additions of different tethered nonaromatic carbon nucleophiles to activated amides $(\mathbf{1} \rightarrow \mathbf{2})$. Additionally, this approach is very interesting because it offers an efficient way to generate various enaminals and enaminones known to be especially useful and versatile intermediates for natural product synthesis. ${ }^{9}$

Before attempting the entire bicyclization $(\mathbf{1} \rightarrow \mathbf{3})$, we opted to set the monocyclization first. To this end, five- and six-membered rings were studied, as they are the most common in the vast majority of alkaloid skeletons. We elaborated a series of model substrates to tackle three different aspects of nucleophilic cyclizations on activated amides: (1) determining the nature of the nucleophiles that could trap the activated amides, (2) comparing five- and sixmembered ring closures, and (3) investigating endo and exo types of cyclization. Both amides and lactams have been looked at, since they generate mono- or bicyclic adducts.

The syntheses of 5- and 6-exo cyclization ${ }^{10}$ substrates started with the ring opening of $\epsilon$-caprolactone (4) with a DIBALH-dibenzylamine complex, ${ }^{11}$ followed by a Swern oxidation to furnish the aldehyde 5 (Scheme 2). The latter was treated either with TBDMSOTf to give the corresponding silyl enol ether $\mathbf{6}$ or with methyl aniline to lead to enamine $7 .^{12}$

Scheme 2. Syntheses of 5- and 6-exo Cyclization Substrates
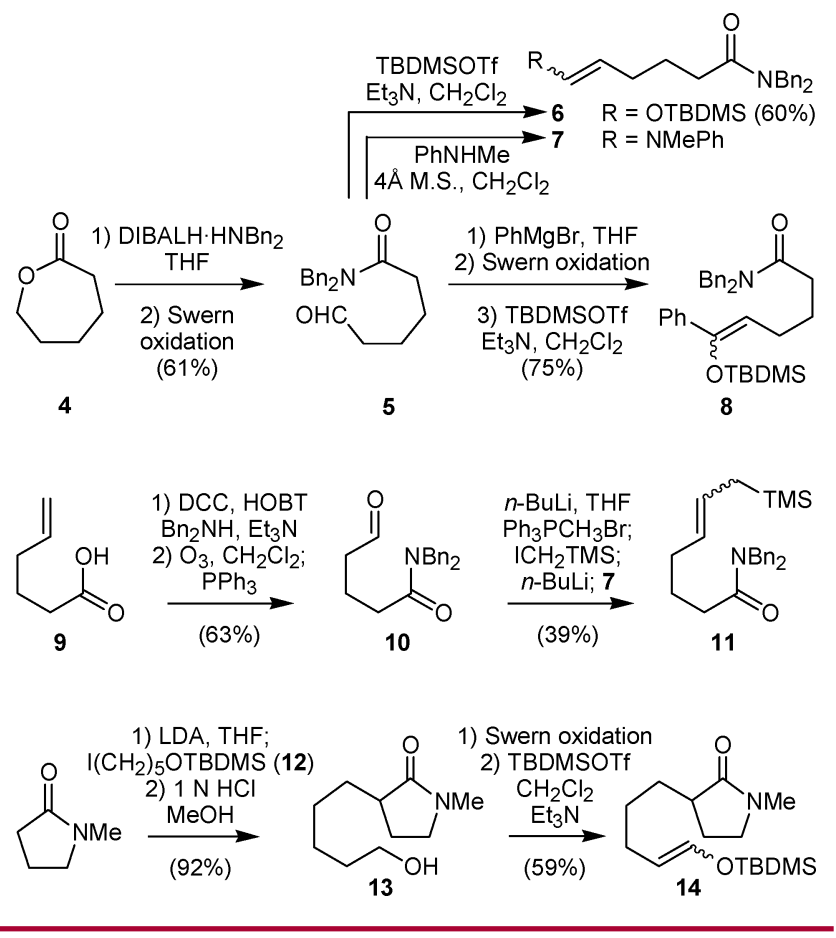

Silyl enol ethers from ketones were also investigated. We prepared the phenyl ketone silyl enol ether $\mathbf{8}^{13}$ by adding phenylmagnesium bromide to the aldehyde $\mathbf{5}$, followed by a Swern oxidation and silylation. We also extended our study to other types of nucleophiles. The allylsilane 11 was thus synthesized in three steps from commercially available carboxylic acid 9, which was coupled with dibenzylamine, ozonized and olefinated. ${ }^{14}$ Finally, a 6-exo cyclization model substrate (14) was built by addition of the iodosilyl ether branch $12^{15}$ to $N$-methylpyrrolidinone enolate, followed by methanolysis of the silyl ether, oxidation and silylation.

Endo types of cyclizations were also investigated. NAlkylation of pyrrolidinone with iodides $\mathbf{1 5}^{16}$ or $\mathbf{1 2}$ followed by the usual sequence furnished the 5-endo and 6-endo cyclization precursors 18 and 19 (Scheme 3). A formamide substrate 22 was also prepared using the usual route.

Scheme 3. Syntheses of 5- and 6-endo Cyclization Substrates
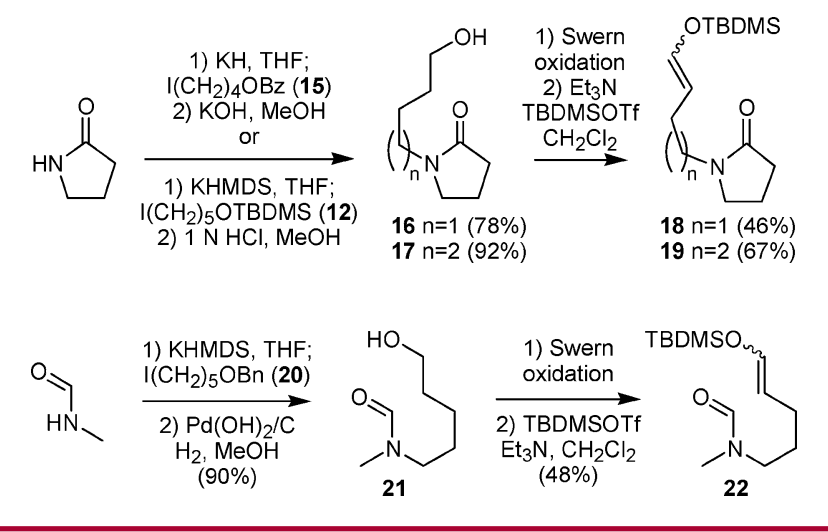

Table 1 shows the variety of nucleophiles that all gave 5-exo cyclizations upon amide activation. Among the as-

(5) For addition of heteroatomic nucleophiles to activated amides, see: (a) Charette, A. B.; Chua, P. Synlett 1998, 163. (b) Charette, A. B.; Chua, P. Tetrahedron Lett. 1997, 38, 8499. (c) Charette, A. B.; Chua, P. J. Org. Chem. 1998, 63, 908. (d) Charette, A. B.; Grenon, M. Tetrahedron Lett. 2000, 41, 1677. (e) Sforza, S.; Dossena, A.; Corradini, R.; Virgili, E.; Marchelli, R. Tetrahedron Lett. 1998, 39, 711. (f) Thomas, E. W. Synthesis 1993, 767. (g) Smith, D. C.; Lee, S. W.; Fuchs, P. L. J. Org. Chem. 1994, $59,348$.

(6) Typically the Bischler-Napieralski cyclization: Bischler, A.; Napieralski, B. Chem. Ber. 1893, 26, 903.

(7) (a) Marson, C. M. Tetrahedron 1992, 48, 3659. (b) Martinez, A. G.; Alvarez, R. M.; Barcina, J. O.; Cerero, S. M.; Vilar, E. T.; Fraile, A. G.; Hanack, M.; Subramanian, L. R. J. Chem. Soc., Chem. Commun. 1990, 1571.

(8) For various amide activation conditions, see references cited in ref $5 \mathrm{~g}$ and in: Kuhnert, N.; Clemens, I.; Walsh, R. Org. Biomol. Chem. 2005, 3, 1694, See also: Nishiyama, H.; Nagase, H.; Ohno, K. Tetrahedron Lett. 1979, 48, 4671. Keck, G. E.; McLaws, M. D.; Wager, T. T. Tetrahedron 2000, 56, 9875 .

(9) For the use of enaminones in natural product synthesis, see: Michael, J. P.; de Koning, C. B.; Gravestock, D.; Hosken, G. D.; Howard, A. S.; Jungmann, C. M.; Krause, R. W. M.; Parsons, A. S.; Pelly, S. C.; Stanbury, T. V. Pure Appl. Chem. 1999, 71, 979.

(10) The use of exo and endo in this manuscript refers to the amide portion of the substratres. For a more appropriate description of cationic cyclizations involving $\pi$-nucleophiles, see: (a) Ben-Ushai, D. J. Chem. Soc. Chem. Commun. 1980, 687. (b) Lochead, A. W.; Proctor, G. R.; Caton, M. P. J. Chem. Soc., Perkin Trans. 1 1984, 2477.

(11) Huang, P.-Q.; Zheng, X.; Deng, X.-M. Tetrahedron Lett. 2001, 42, 9039.

(12) Enamine 7 was unstable and had to be used without further purification in the cyclization step. 
Table 1. 5-exo Cyclizations of Various Tethered Nucleophiles on Activated $N, N$-Dibenzylamides

entry

${ }^{a}$ Activation conditions: (A) $\mathrm{Tf}_{2} \mathrm{O}$ (1.1 equiv), 2,6-di-tert-butyl-4methylpyridine (1.1 equiv), $\mathrm{CH}_{2} \mathrm{Cl}_{2}, 0^{\circ} \mathrm{C}, 20 \mathrm{~min}$; (B) $\mathrm{Tf}_{2} \mathrm{O}$ (2.5 equiv), ${ }^{i} \mathrm{Pr}_{2} \mathrm{NEt}$ (3.0 equiv), $\mathrm{CHCl}_{3}, 0{ }^{\circ} \mathrm{C}, 20$ min. ${ }^{b}$ Yields of isolated product. ${ }^{c}$ Pyr $\cdot \mathrm{HF}, 0{ }^{\circ} \mathrm{C}, 20 \mathrm{~min} .{ }^{d}$ Concentrated and then diluted in $1 \mathrm{~N} \mathrm{NaOH}$, THF, $25{ }^{\circ} \mathrm{C}$, overnight. ${ }^{e} \mathrm{NaBH}(\mathrm{OAc})_{3},-78{ }^{\circ} \mathrm{C}$ to $\mathrm{rt}$, overnight. An inseparable 94:6 mixture of $Z / E-\mathbf{2 5}$, respectively, as established by nOe. ${ }^{f}$ Concentrated and then purified directly.

sortment of amide activating agents we surveyed, triflic anhydride $\left(\mathrm{Tf}_{2} \mathrm{O}\right)$ is the reagent of choice and gave reproducible results with all of the substrates tested. $\mathrm{POCl}_{3}$ and triphosgene led to the cleavage of the silyl enol ethers (the corresponding aldehydes were recovered), whereas TMSOTf and TFAA gave undesired side products.

The amide activation with $\mathrm{Tf}_{2} \mathrm{O}$ was always completed within $20 \mathrm{~min}$ at $0{ }^{\circ} \mathrm{C}^{17}$ in the presence of 2,6-di-tert-butyl4-methylpyridine or di-isopropylethylamine to prevent the generation of triflic acid. These rather mild conditions were tolerated by TBDMS enol ethers from aldehydes or from ketones (Table 1, entries 1 and 2 respectively), as well as by allylic trimethylsilane (entry 3 ) and $N$-methyl- $N$-phenylenamine (entry 4). The aldehyde TBDMS enol ether $\mathbf{6}$ gave the highest yield, cyclizing in $85 \%$ yield (entry 1 ). The phenyl ketone analogue $\mathbf{8}$ was also successfully cyclized even though the yields were slightly lower (entry 2 versus 1 ), presumably because of a higher steric congestion developed during cyclization. When the allylsilane 11 was cyclized (entry 3$)$, a reductive quench $\left(\mathrm{NaBH}(\mathrm{OAc})_{3}\right)$ was necessary, since the iminium ion resulting from the cyclization was not stable. ${ }^{18}$ An overall yield of $81 \%$ was obtained for the cyclization-reduction sequence.

Finally, upon activation of amide 7, we were able to isolate and characterize the vinylogous amidinium ion $\mathbf{2 6}$. As the conversion of aldehyde 5 to enamine 7 was estimated at $80-$ $85 \%{ }^{17}$ and the overall yield of $\mathbf{2 6}$ from aldehyde $\mathbf{5}$ is $74 \%$,

(13) The phenyl was essential since we had to control on which side the ketone would enolize during the final silyl enol ether preparation.

(14) Fleming, I.; Paterson, I. Synthesis 1979, 446.

(15) Deng, Y.; Salomon, R. C. J. Org. Chem. 1998, 63, 3504.

(16) Ott, M. M.; Little, R. D. J. Org. Chem. 1997, 62, 1610.

(17) As observed by ${ }^{1} \mathrm{H}$ NMR.

(18) The resulting iminium ion after the cyclization was not conjugated with the remaining alkene according to ${ }^{1} \mathrm{H}$ NMR spectroscopy, presumably because of high allylic strain resulting from conjugation. the cyclization yield from $\mathbf{7}$ to $\mathbf{2 6}$ is evaluated at around $85 \%$. This means that silyl enol ethers, allylsilanes, and enamines add to activated amides with comparable ease and yields under mild conditions.

The $\mathrm{Pyr} \cdot \mathrm{HF}$ (or $\mathrm{NaOH}$ as found in Table 2 ) $^{19}$ quench is essential to cleave the TBDMS group still attached to the

Table 2. 5-exo, 6-exo, 5-endo, and 6-endo Cyclizations of Tethered TBDMS Enol Ethers on Activated Amides and Lactams $^{a}$

entry

${ }^{a}$ Activation conditions: $\mathrm{Tf}_{2} \mathrm{O}$ (1.1 equiv), 2,6-di-tert-butyl-4-methylpyridine (1.1 equiv), $\mathrm{CH}_{2} \mathrm{Cl}_{2}, 0{ }^{\circ} \mathrm{C}, 20 \mathrm{~min} .{ }^{b}$ Yields of isolated product. ${ }^{c}$ Concentrated and then diluted in $1 \mathrm{~N} \mathrm{NaOH}$, THF, $25{ }^{\circ} \mathrm{C}$, overnight. ${ }^{d}$ $\mathrm{Pyr} \cdot \mathrm{HF}, 0{ }^{\circ} \mathrm{C}, 20 \mathrm{~min}$.

carbonyl when enol ethers were cyclized. ${ }^{17}$ Such a detail is highly important: regardless of the tethered nucleophile used, there is always an activated species in solution before quenching the reaction.

A study comparing ring sizes as well as endo and exo cyclizations was also performed with aldehyde silyl enol ethers. The 5-exo and the 6-endo cyclizations of alkylamides 6 and 19 gave the highest yields (Table 2, entries 1 and 4 respectively), followed by the 6-exo cyclization of $\mathbf{1 4}$ (entry 2). As expected, the 5-endo cyclization of substrate $\mathbf{1 8}$ was quite difficult, which is in accordance with the Baldwin rules, ${ }^{10,20}$ but nonetheless successful (35\% yield, entry 3 ). Surprisingly, the 6-endo cyclization of the less congested iminium ion upon formamide $\mathbf{2 2}$ activation gave lower conversion than the cyclization of pyrrolidinone 19. This could be due to a lower stability of the iminium ion derived from 22.

In summary, we demonstrated for the first time that activated amides could be trapped with various tethered nonaromatic carbon nucleophiles very efficiently. The resulting enaminals cover a wide range of skeletons of various

(19) The quench procedure has a crucial influence on the cyclization yield that we do not yet fully understand. Investigation in that direction is underway and will be published in due course.

(20) (a) Baldwin, J. E. J. Chem. Soc., Chem. Commun. 1976, 734. (b) Baldwin, J. E.; Cutting, J.; Dupont, W.; Kruse, L.; Silberman, L.; Thomas, R. C. J. Chem. Soc., Chem. Commun. 1976, 736. 
ring sizes containing either an endo- or exo-cyclic nitrogen. The alkenes of the enaminals are either tetra- or trisubstituted depending on whether the initial amide carbonyl was alkylated or not. Ultimately, upon double nucleophilic additions, related systems (such as $\mathbf{1}$, Scheme 1) could lead to quaternary or tertiary centers $\alpha$ to nitrogen (such as $\mathbf{3}$ ). Moreover, we also demonstrated that the intermediates obtained before quenching of the cyclization reaction were still iminium ions, thus conferring optimistic perspective to our planned biscyclization with two nucleophilic moieties tethered to the amide. Work in that direction is currently in progress.
Acknowledgment. We thank Ghislain Boucher for his early work on this project. This research was supported by the Natural Science and Engineering Research Council (NSERC) of Canada, FQRNT (Québec), the Canadian Fund for Innovation (CFI), and the Université de Sherbrooke. NSERC doctoral fellowship to R.L.-G. and NSERC predoctoral fellowship to F.B. are also gratefully acknowledged.

Supporting Information Available: Experimental procedures and ${ }^{1} \mathrm{H}$ and ${ }^{13} \mathrm{C}$ NMR spectra for compounds 5-8, $\mathbf{1 0}, \mathbf{1 1}, \mathbf{1 3}, \mathbf{1 4}, \mathbf{1 6}-\mathbf{1 9}$, and $\mathbf{2 1 - 3 0}$. This material is available free of charge via the Internet at http://pubs.acs.org.

OL0516519 